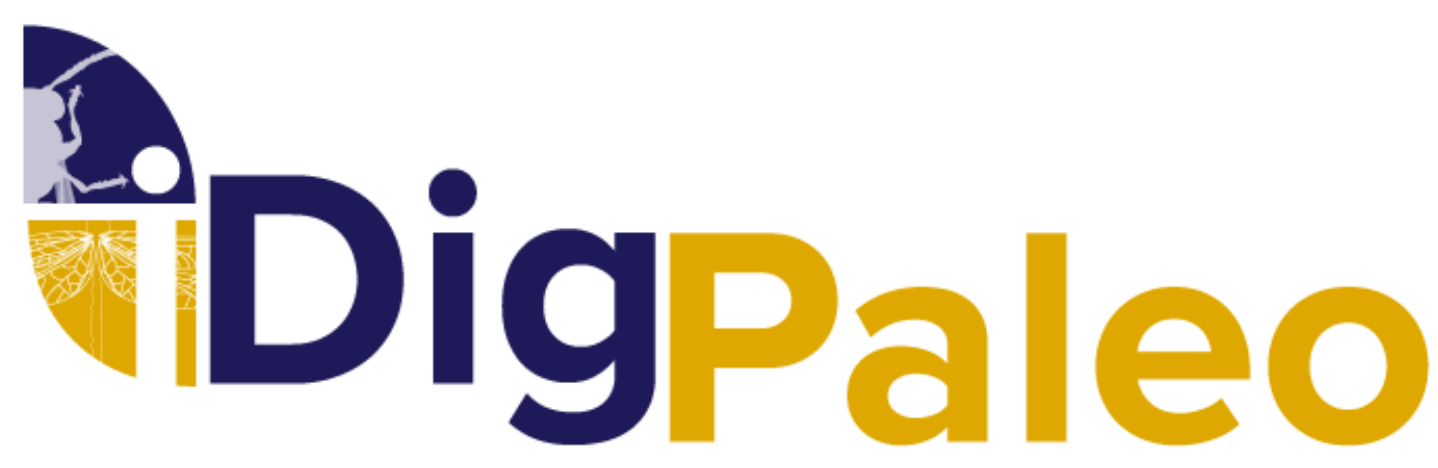

\title{
Education and outreach using digitized museum specimens
}

Susan Butts, Talia Karim, and Christopher Norris 

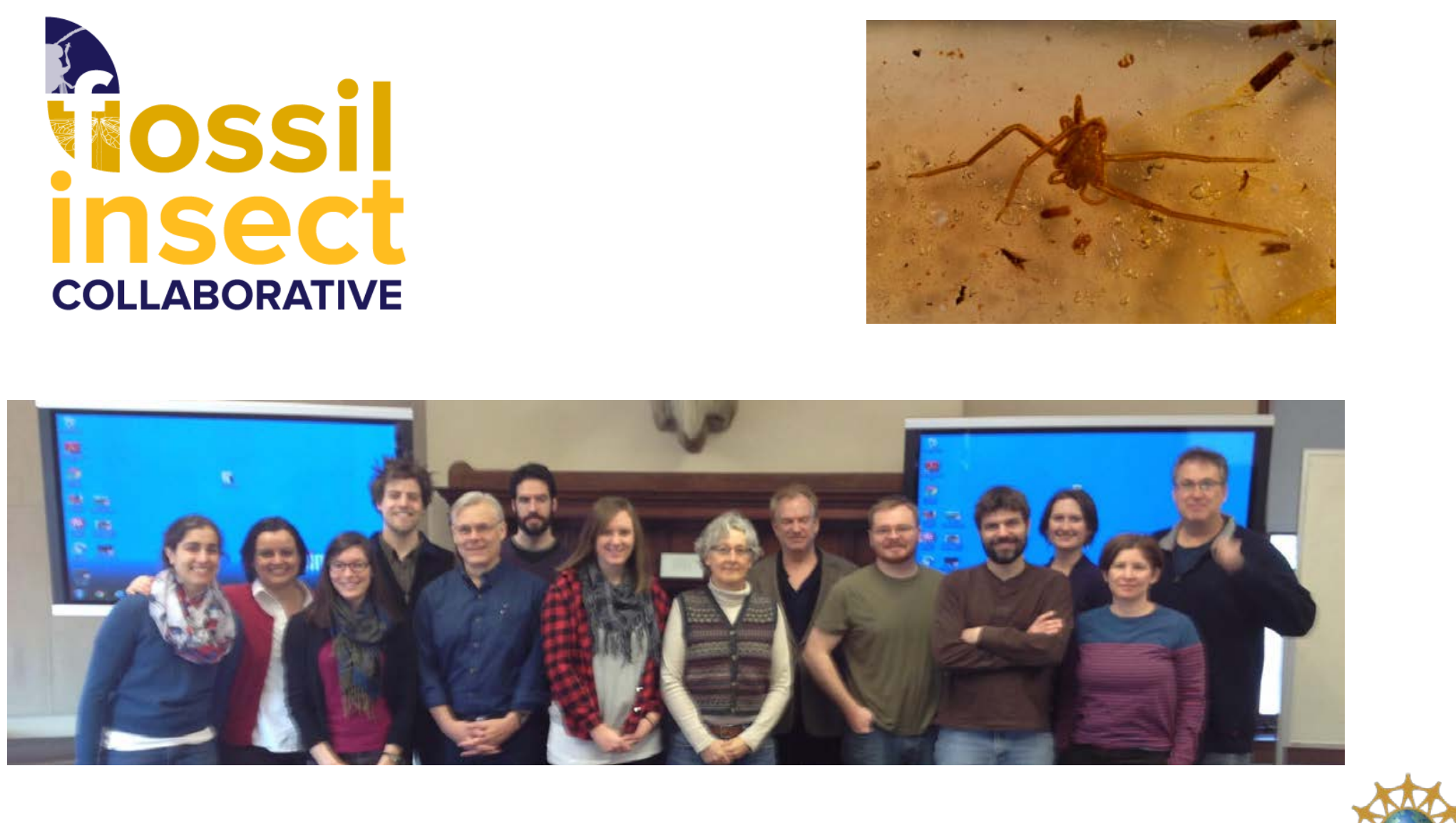
Take our 30-second survey

The U.S. National Science Foundation and iDigBio are required to collect information on use of digitized collections-based specimen data. Please portal. Sustainability of the national digitization effort depends on evidence of data use! Maybe later.

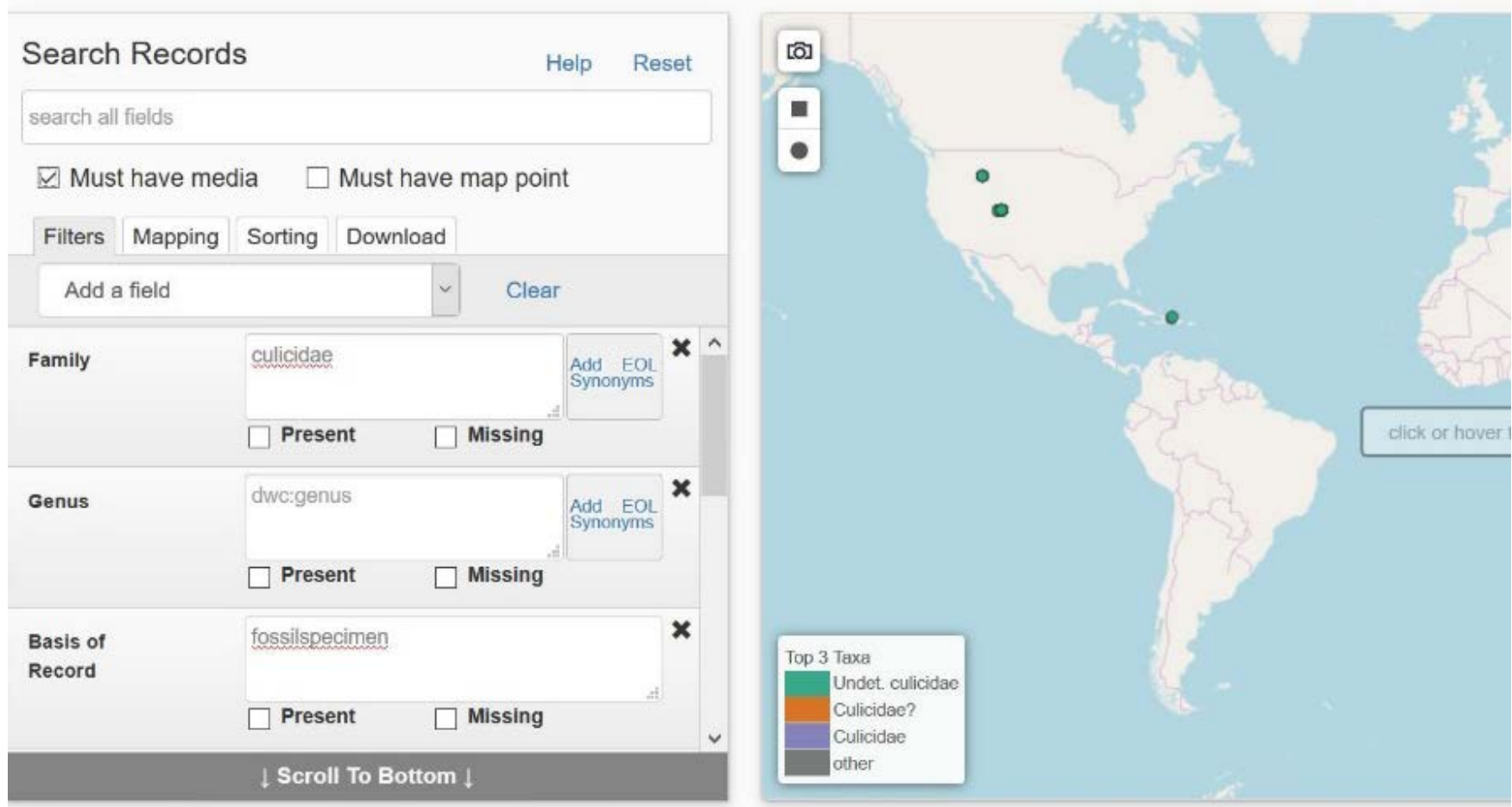



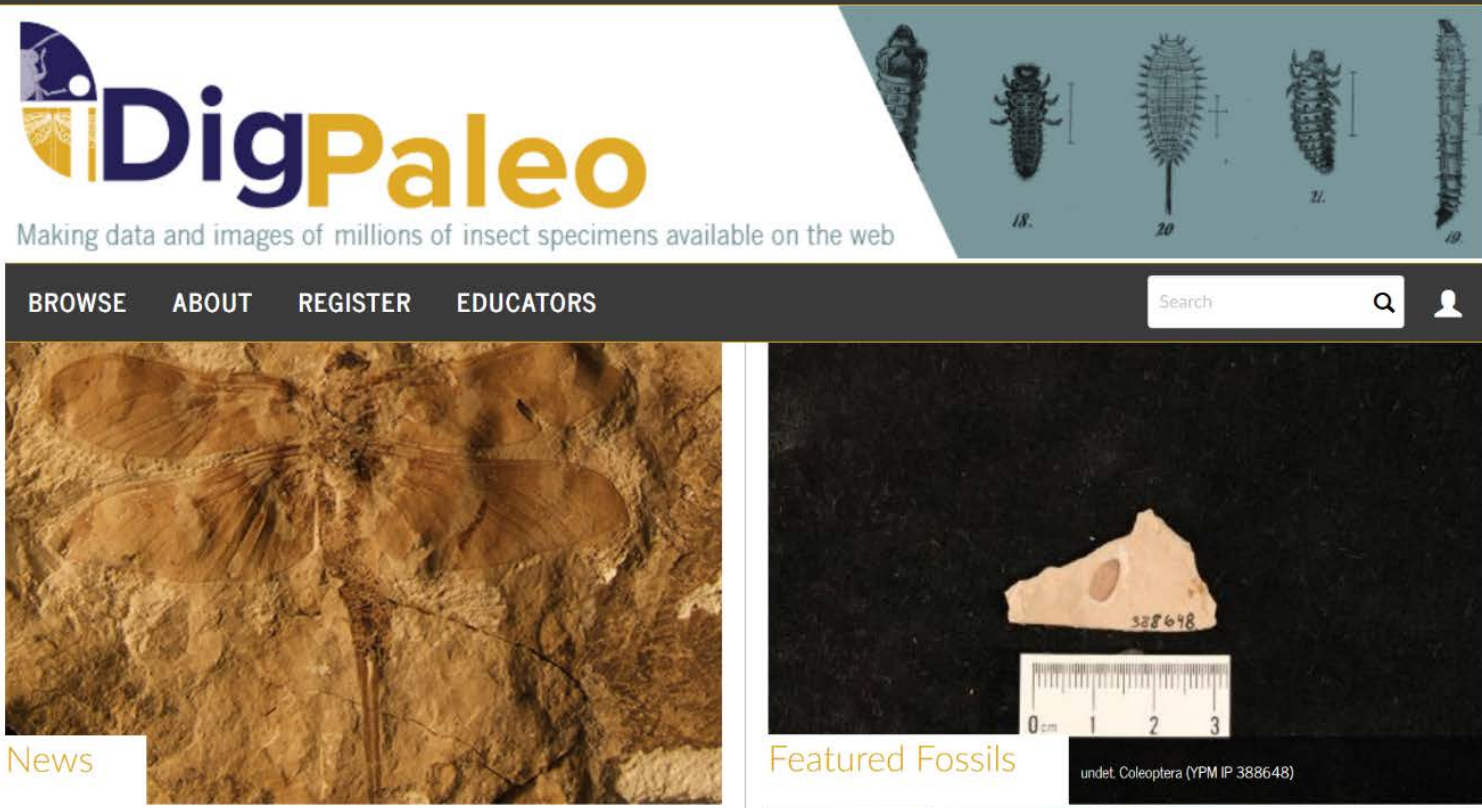

Fossil Insect Collaborative @ GSA 2016

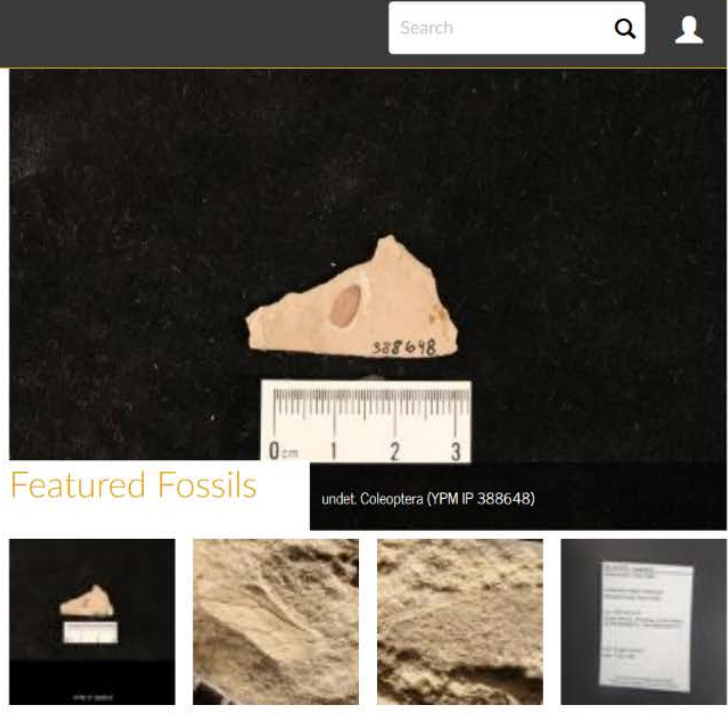

Tweets by @FossillnsectTCN

Alossil Fossil Insect TCN

(nisocec @FossillnsectTCN

Need some weekend reading? Check out this new paper on fossil rove beetles. \#FossilFriday

O $[\rightarrow$

May 5, 2017
Latest Comments

66 Microgastrinae

- KATJA SELTMANN

View USNM Paleobiology PAL580913.11705449

66 cute bee

-SUSAN BUTTS

View YPM IP 560556
Featured Institution

Yale 


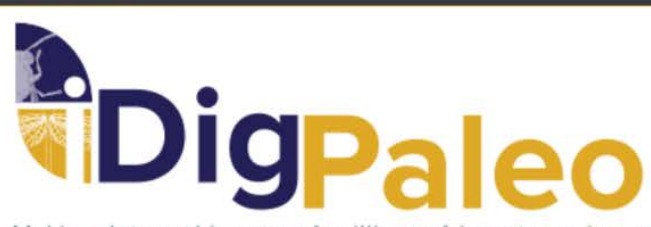

Making data and images of millions of insect specimens available on the web
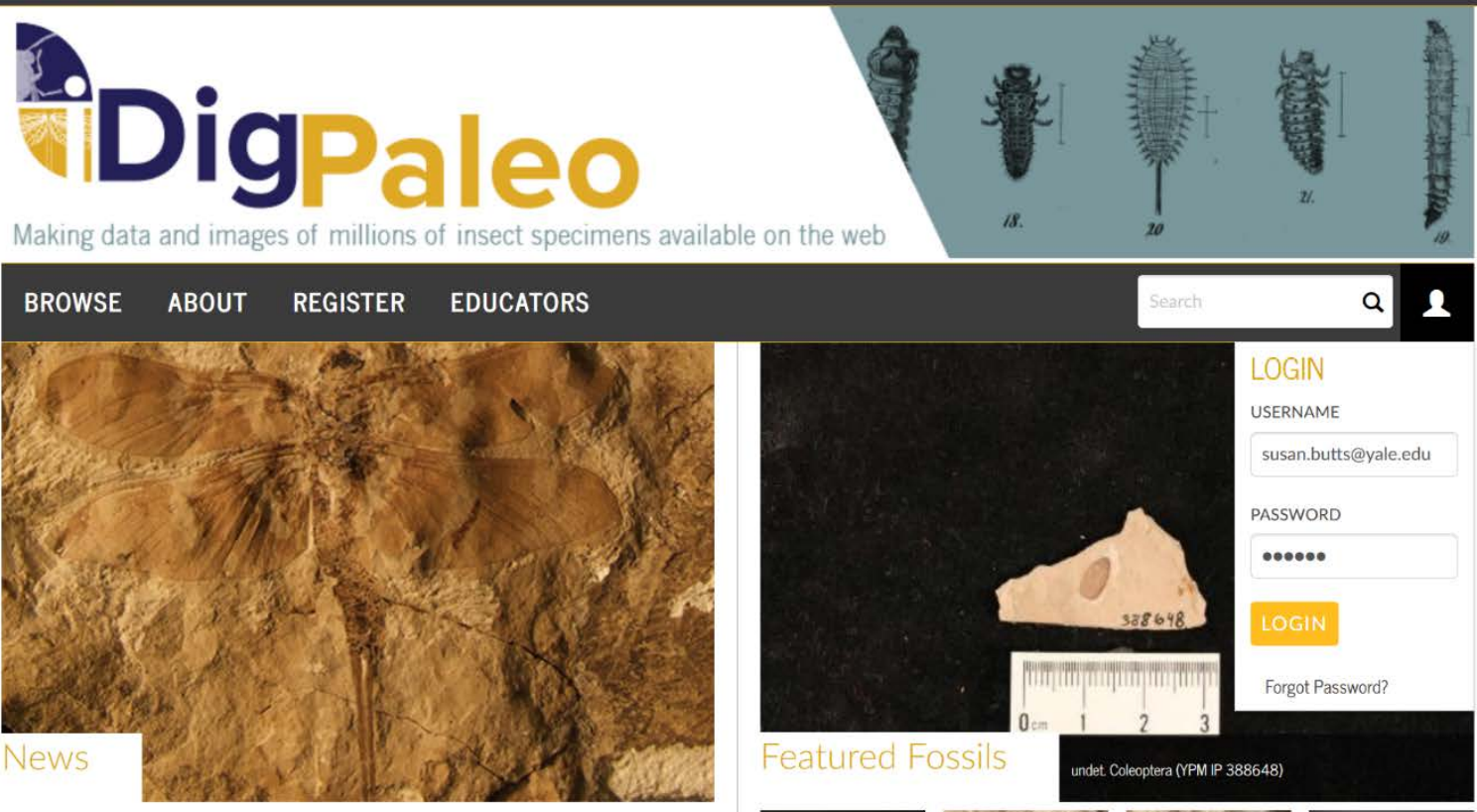

Fossil Insect Collaborative @ GSA 2016

More
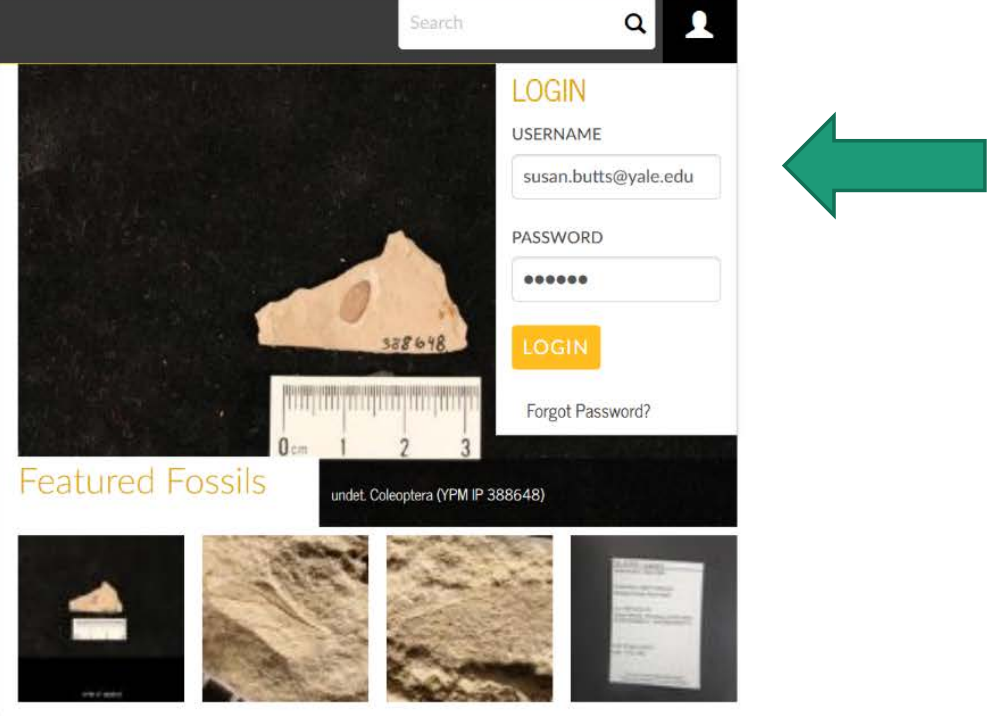

register

Latest Comments

Tweets by @FossillnsectTCN

fiossil Fossil Insect TCN

1nsecy @issillnsectTCN

Need some weekend reading? Check out this new

paper on fossil rove beetles. \#FossilFriday

C $[\rightarrow$

May 5, 2017

Fossil Insect TCN
Featured Institution

Yale 
Making data and images of millions of insect specimens available on the web

\begin{tabular}{|l|l|l|}
\hline BROWSE ABOUT REgISTER EDUCATORS & Search $\mathbf{Q}$ \\
\hline
\end{tabular}

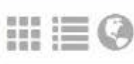

FILTERBY

FOSSIL/MODERN 6

COMMON NAME 6

PERIOD 9

CONTINENT 19

Genus $\mathbf{B}$

FOSSIL DEPOST 6

LOCALTY 8

SOURCE 1

default browse
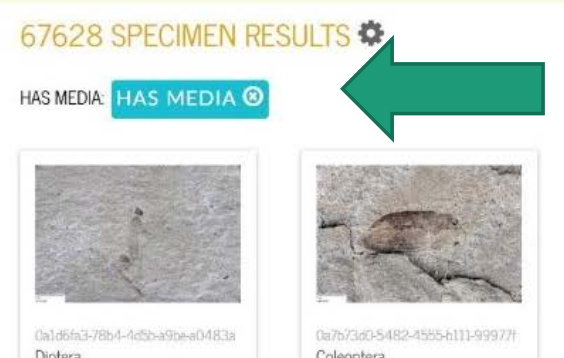

Diptera
Cornoon Nome True flies. Mos.
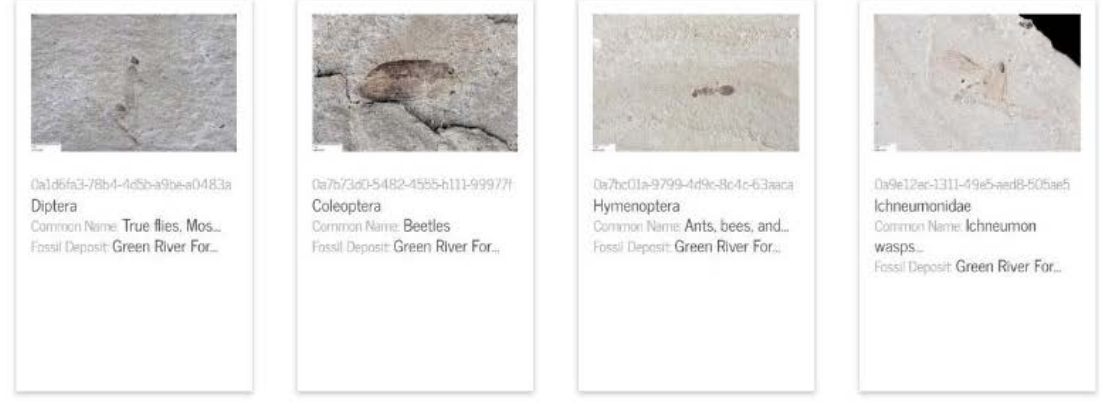

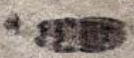

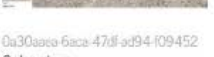

Coleoptera

Cotrmon Nere Beeties

Fossil Deposit Green River For-

Coleoptera
Commorn Nane Beetles
fossil Deposit Green River For.
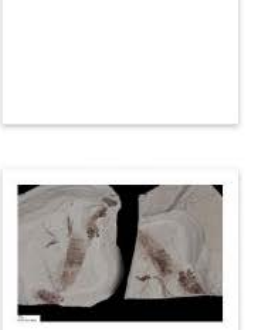

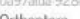

Orthoptera

Fossil Deposit Green River For.

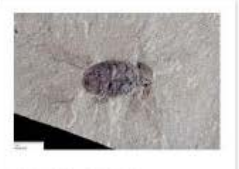

0.3329 .360044489

Curculionidae

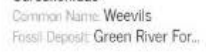

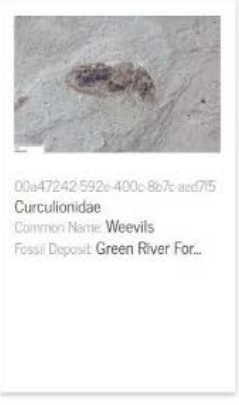


Making data and images of millions of insect specimens available on the web

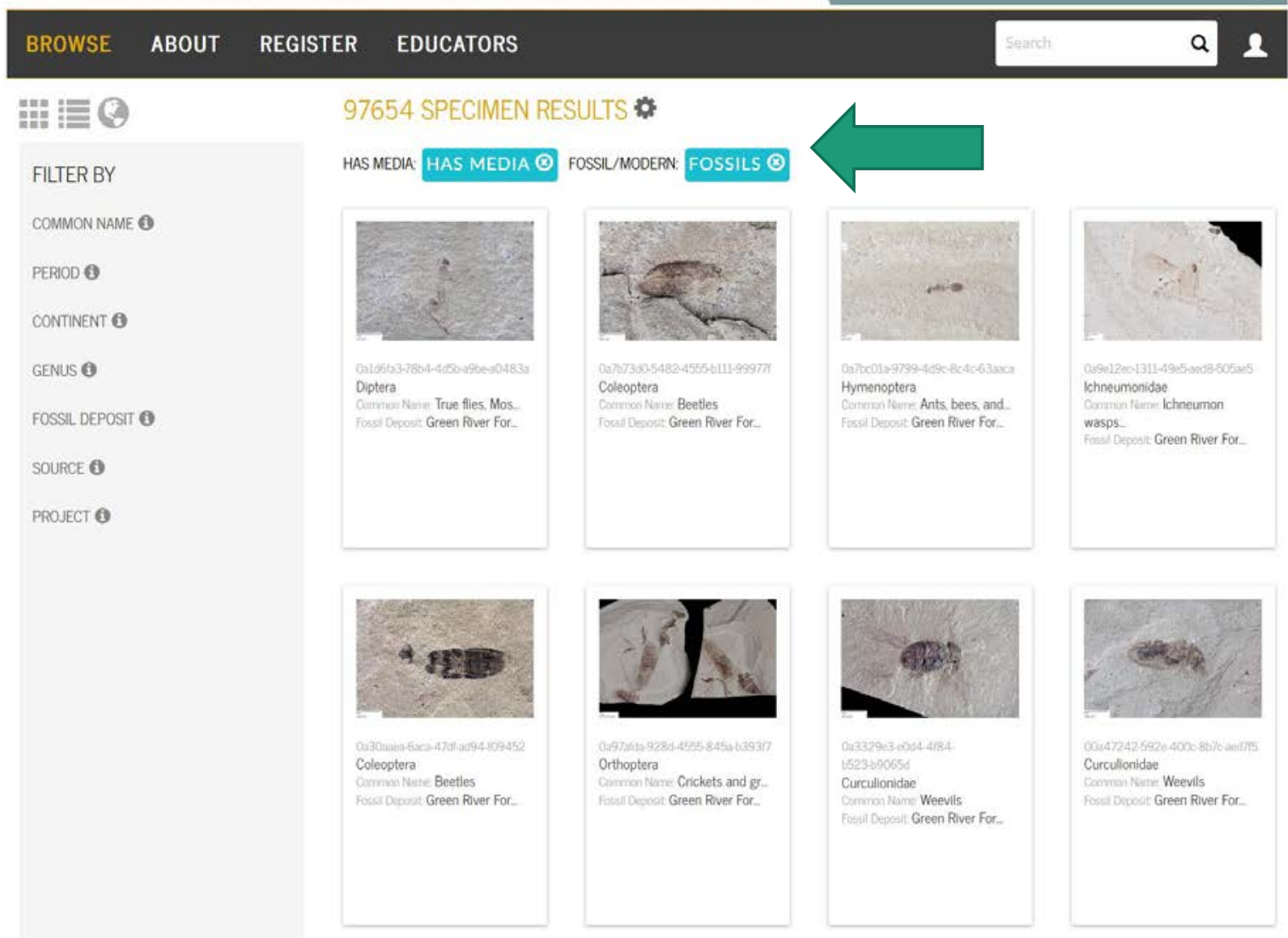

\section{filtered query}




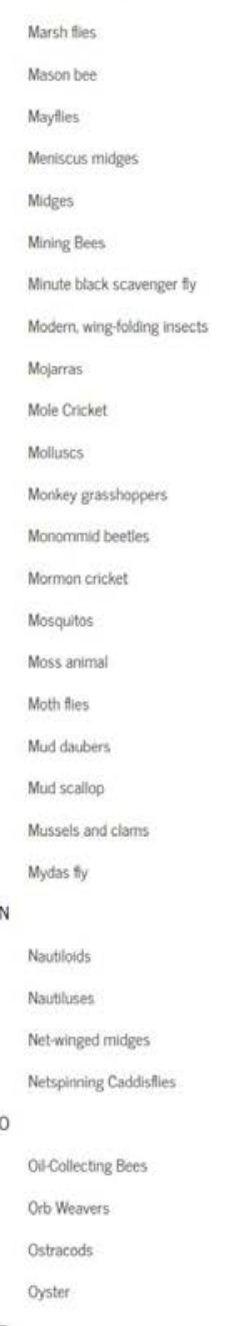

\section{eo}

sect specimens available on the web

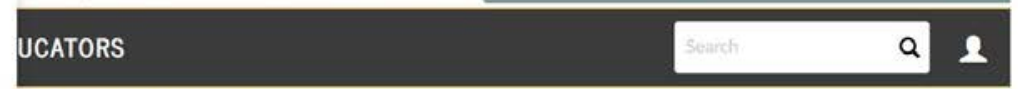

SPECIMEN RESULTS *

HAS MEDIA $\bigcirc$
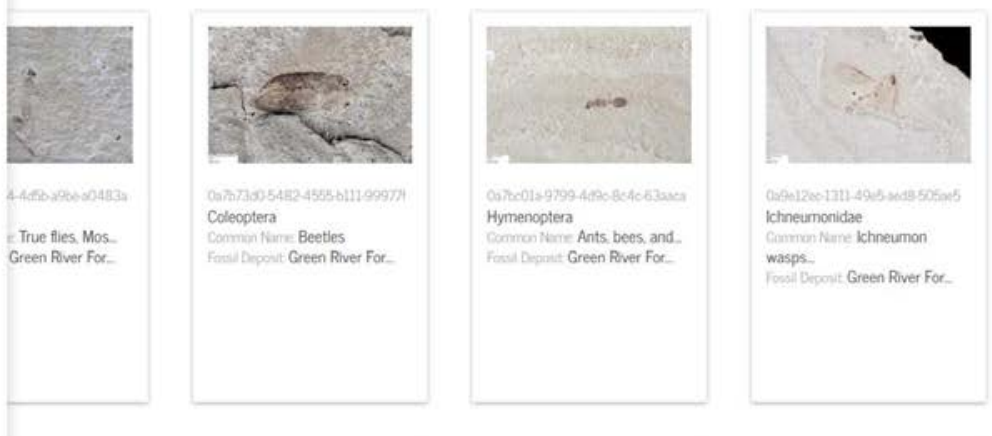

common
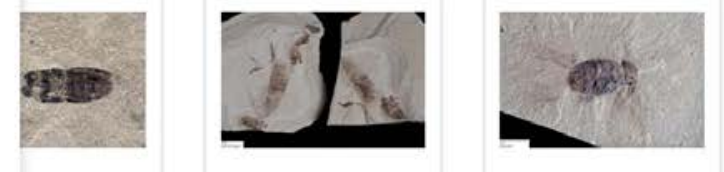

Orthoptera Crickets and

Beetles

Green River Fox-

Fomal Depant Green River For.

Curculionidae

Cosuron Nere Weevils

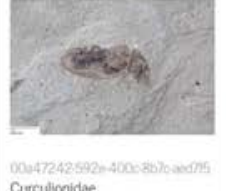

Curcullonidoe ${ }^{2}$ Wevils

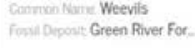
names 


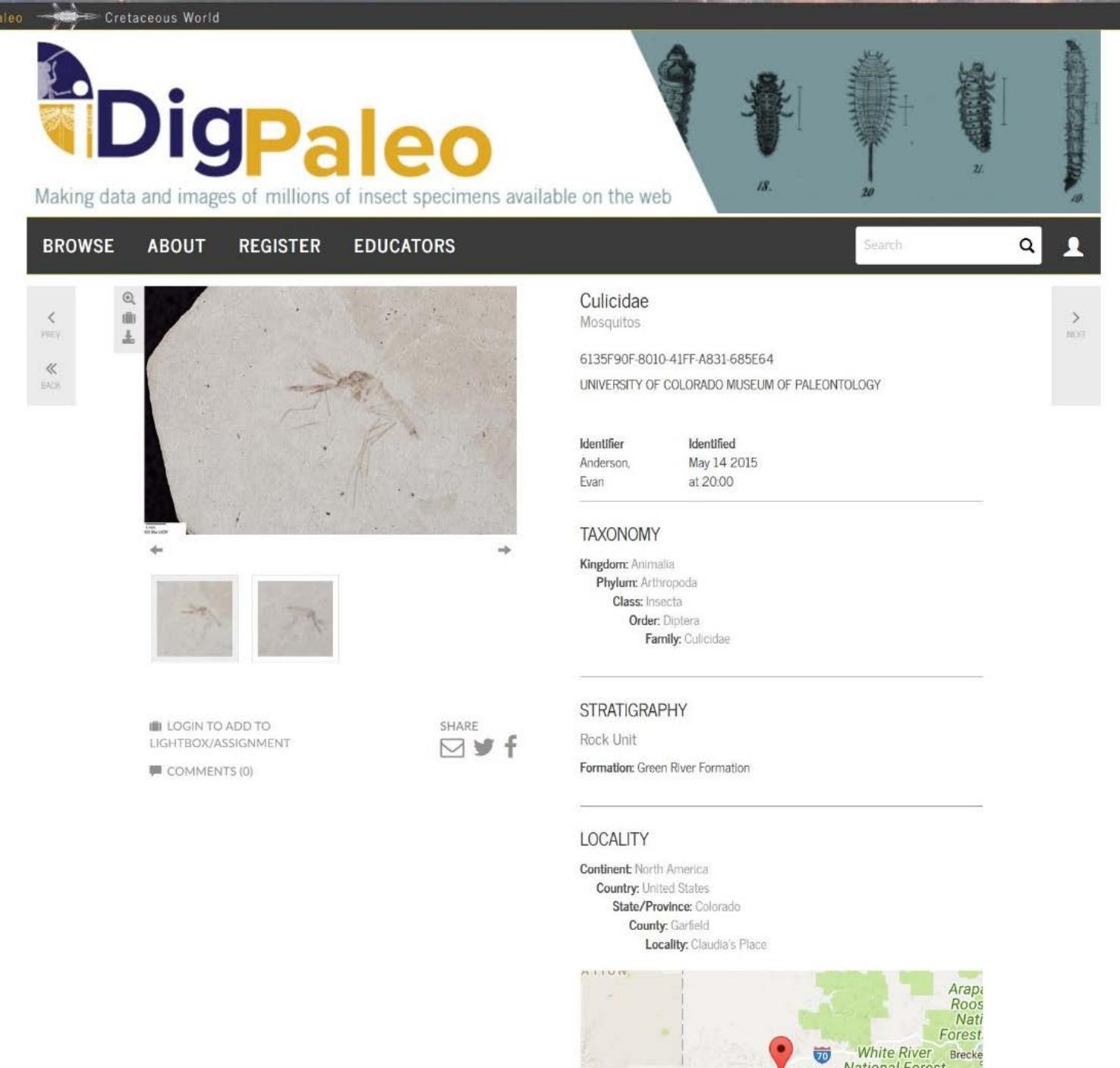




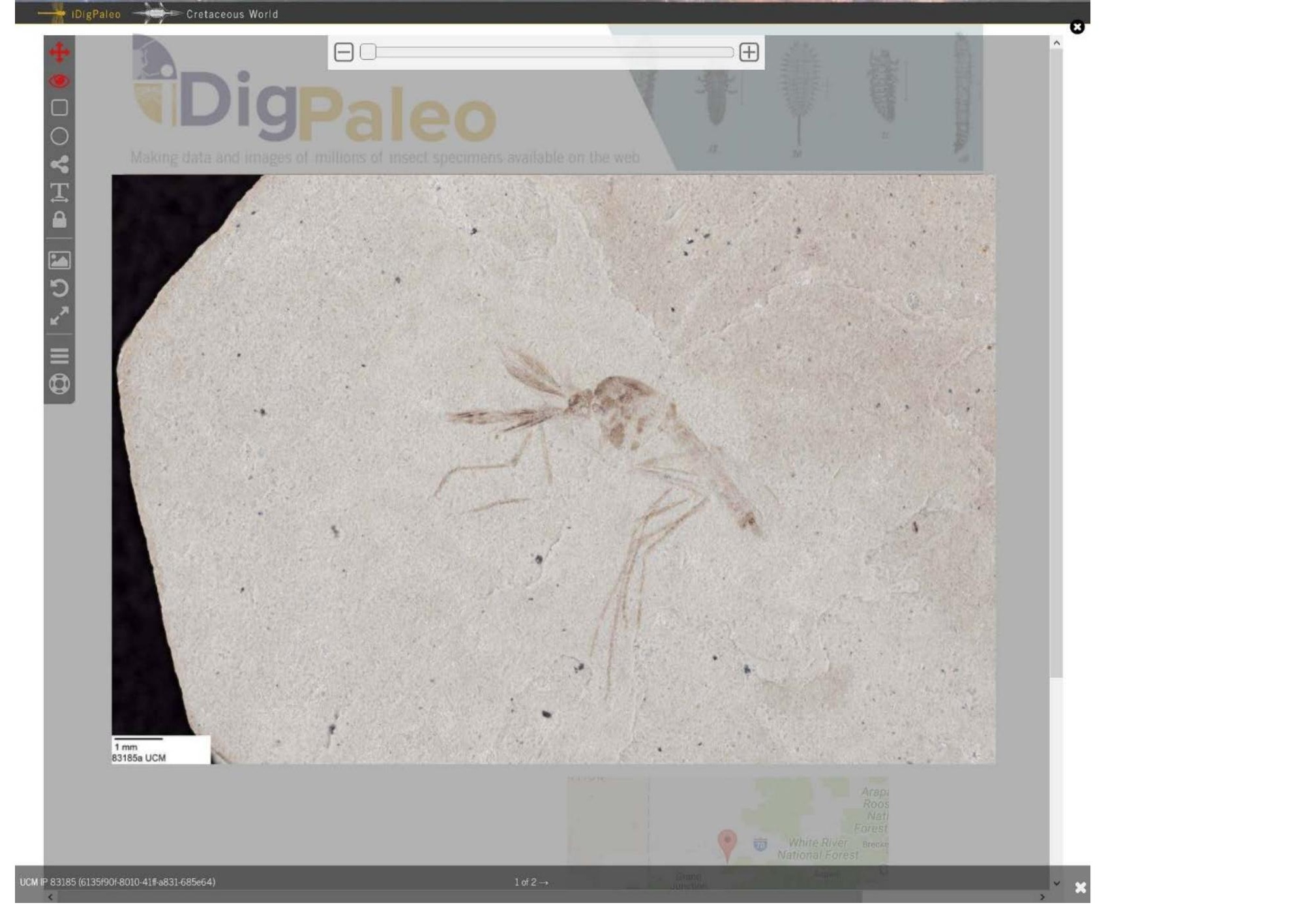




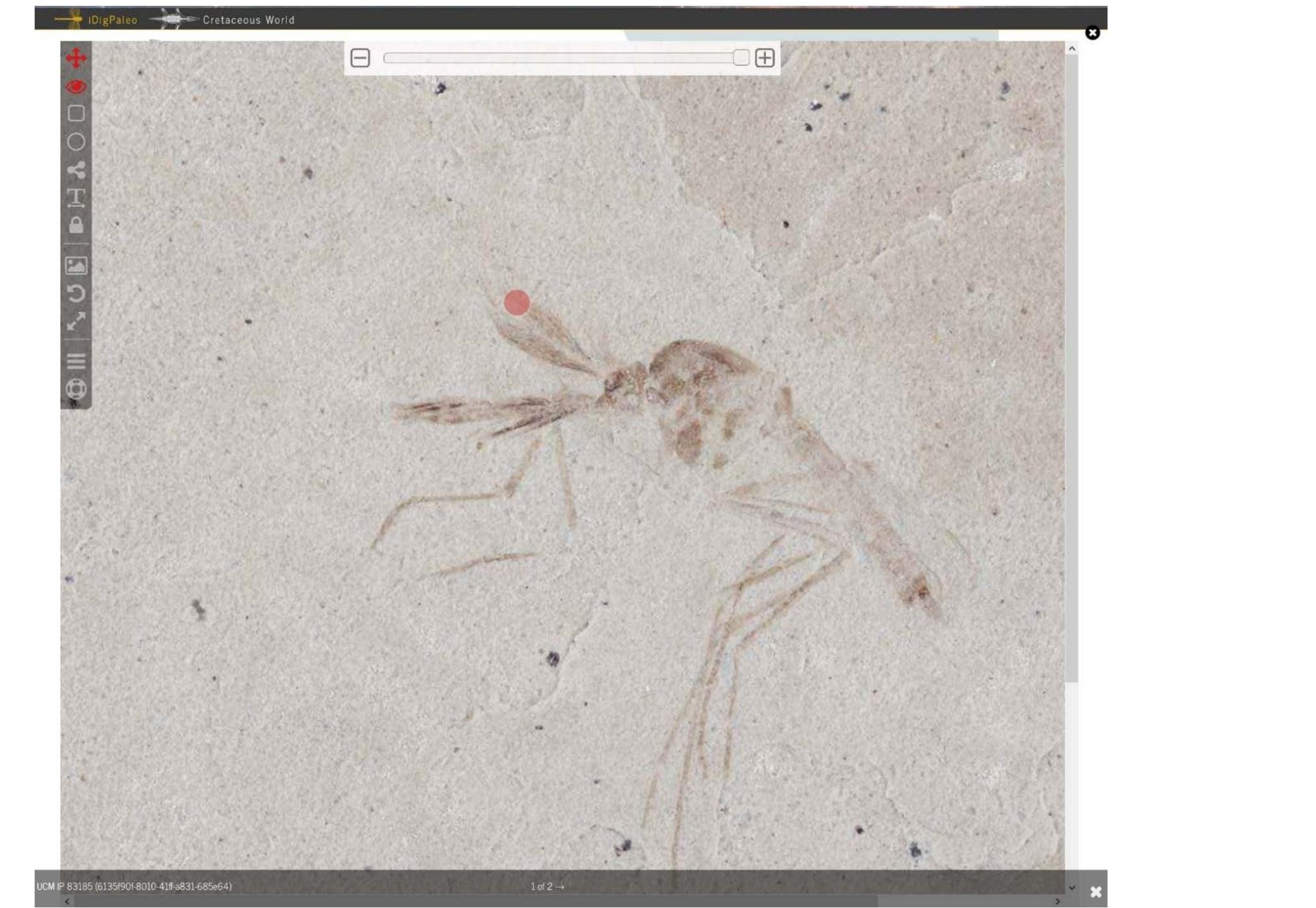


measure/

set scale
$\Rightarrow$ IDigPaleo $\Rightarrow$ Cretaceous World

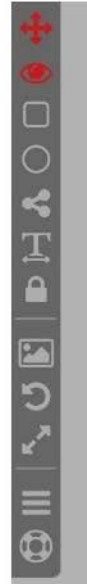

Dig

$\boxminus$

$\boxplus$

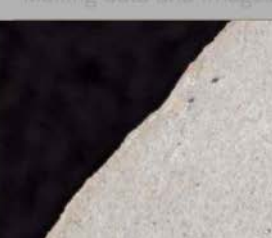

Need

A scale must be set for this image before measurements
can be evaluated

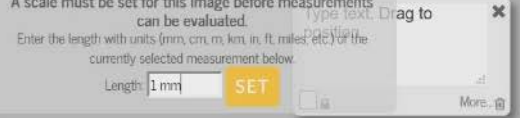

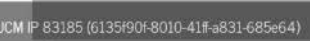


- DigPaleo $=$ cretaceous World

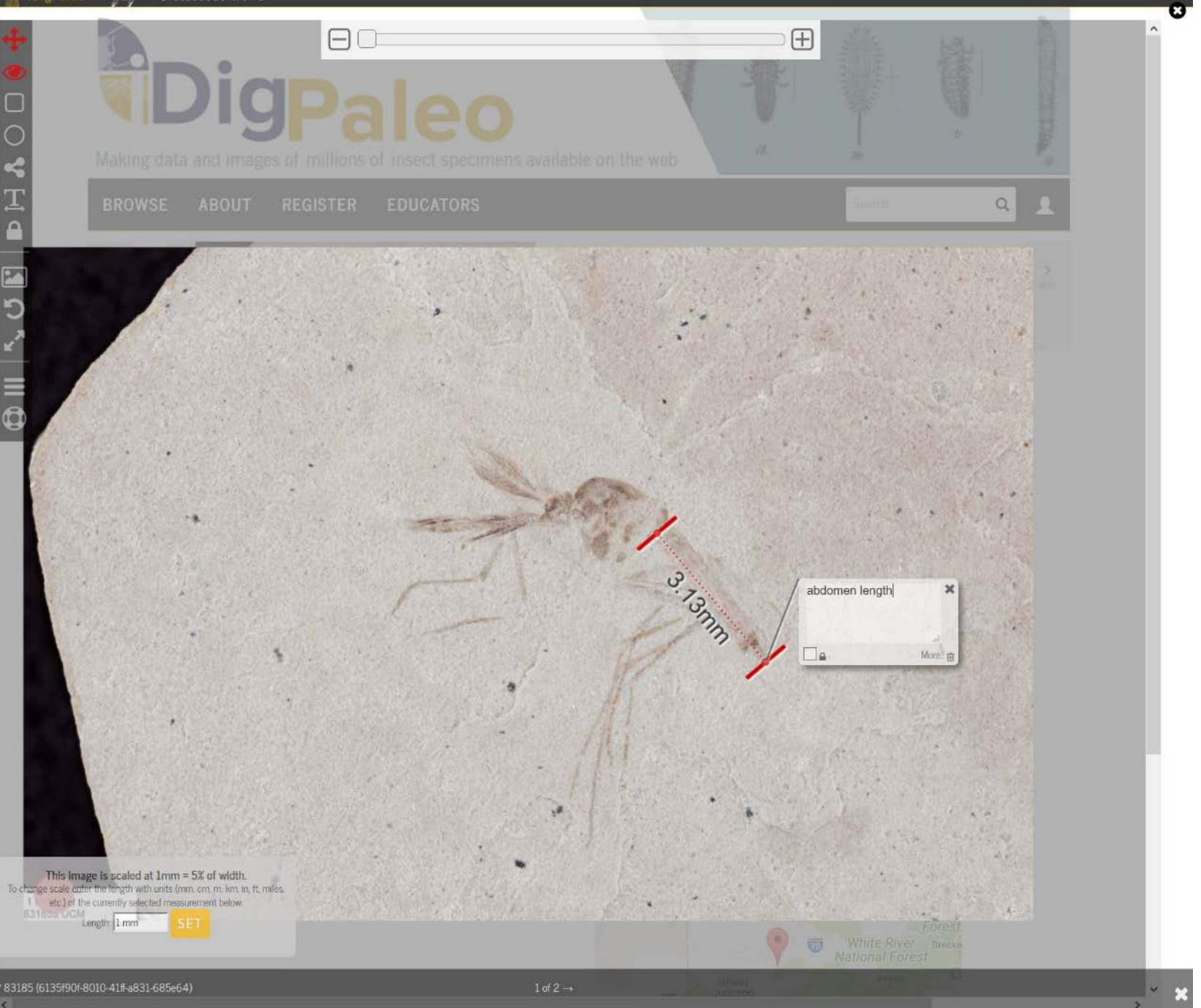

measure/ annotate 
$\Longrightarrow$ DigPaleo $\Rightarrow$ cretaceous World

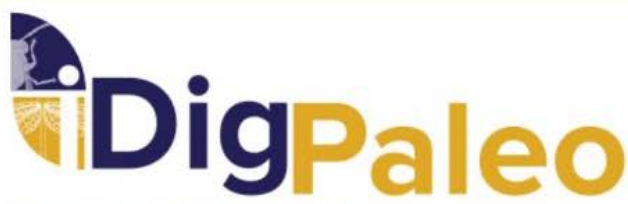

Making data and images of millions of insect specimens available on the web

BROWSE ABOUT REGISTER EDUCATORS
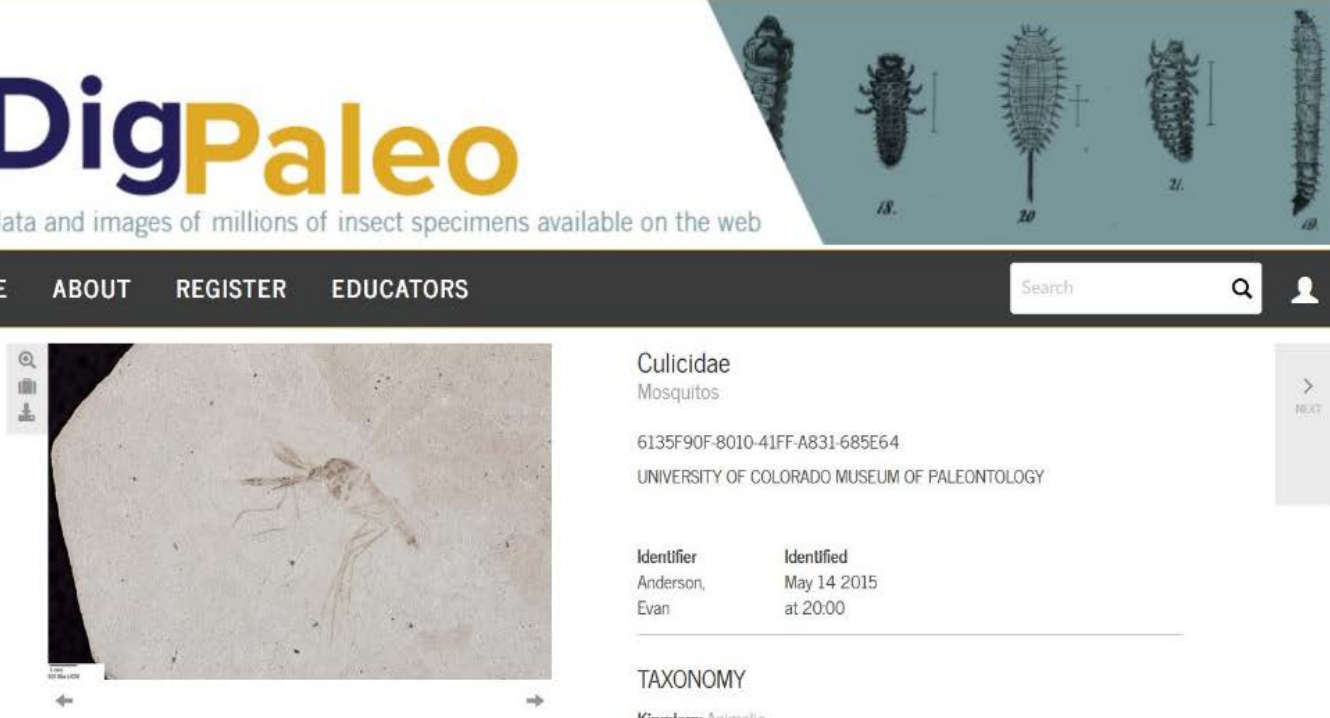

Culicidae

$$
\text { Mosquitos }
$$

6135F90F-8010-41FF-A831-685E64

UNIVERSTYY OF COLORADO MUSEUM OF PALEONTOLOGY

Identifier Identified

Anderson. May 142015

Evan

TAXONOMY

Kingdorn: Animalia

Phylumr Arthropoct

Class: insecta

Family. Culicida:

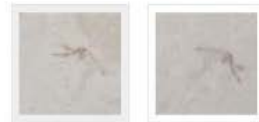

May 1420
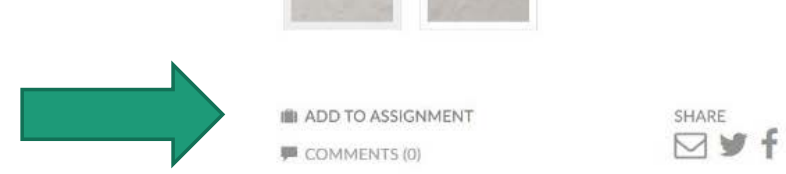

STRATIGRAPHY

Rock Unit

Formation: Green River Formation

$$
\begin{aligned}
& \text { LOCALITY } \\
& \text { Continent North America } \\
& \text { Country: United States } \\
& \text { State/Province: Colorato } \\
& \text { County. Garfield }
\end{aligned}
$$


$\longrightarrow$ IDigPaleo $\rightarrow$ Cretaceous World

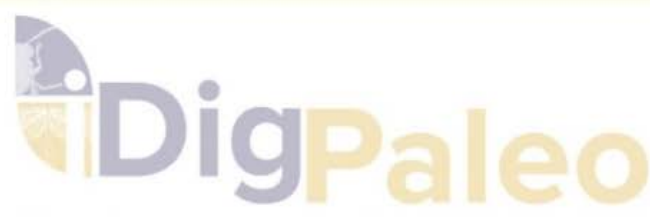

Making data and images of millions of insect specimens avallable on the web

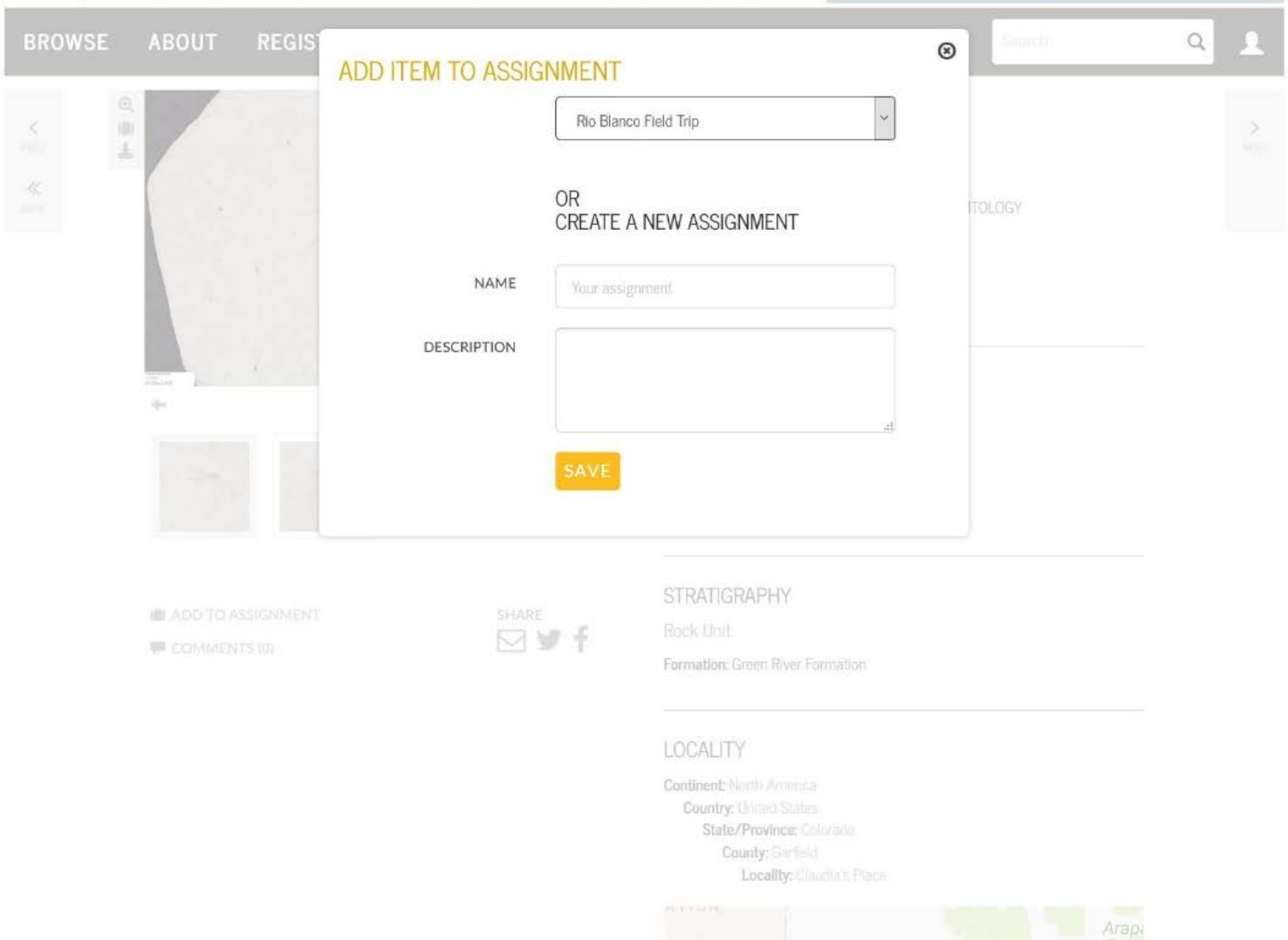

\section{curate}


Making data and images of millions of insect specimens available on the web

\section{CLASSROOM}

ANTS

ant records with images

둔

i)
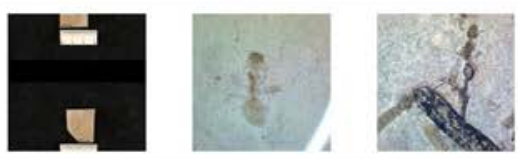

Itens 11 Comments 1 Responses 0

RIO BLANCO FIELD TRIP

Geology 540 Green River Field Trip
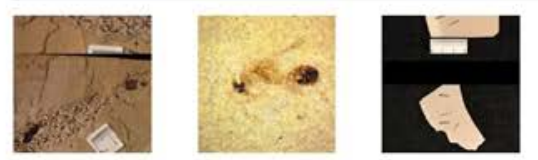

Items 4 Comments 0 Responses

C2 \&

\section{NEW ASSIGNMENT}

\section{Your Groups}

Groups are an easy way to share your

assignments with your classes. Simply make a group, and use the Share Assignment button to link your assignments with groups. Individual students can jain the group by following the group's Urt

\section{NEW GROUP}

teacher workshop

อ 


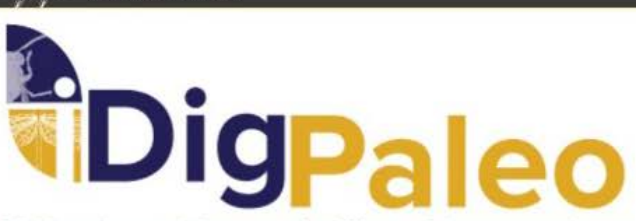

Making data and images of millions of insect specimens available on the web
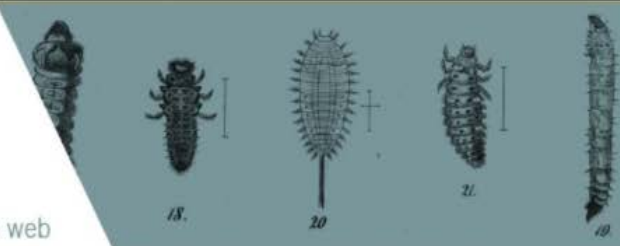

"«x RIO BLANCO FIELD TRIP (4 Items)

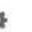

Geology 540 Green River Field Trip

All assignments

Edit Name/Description Share Assignment
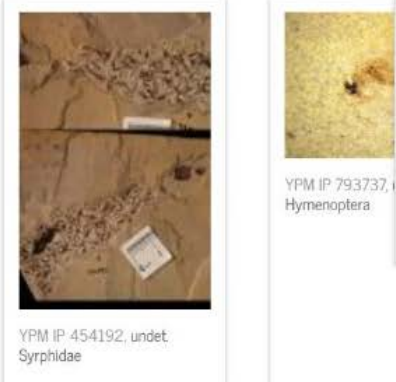

Manage Assignment Access

Start presentation

New Assignment

New User Group

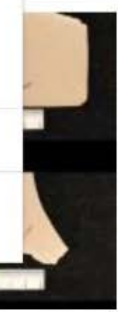

i

\section{create}


UCM IP 83185

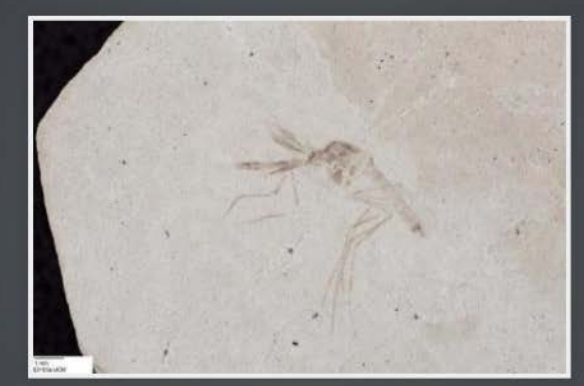

6135F90F-8010-41FF-A831-685E64 
How we got here...

\section{A database is not enough...}

- common names

- curated collections (assignments)

- background materials

- videos from collections/field

- lesson plans based on NGSS+

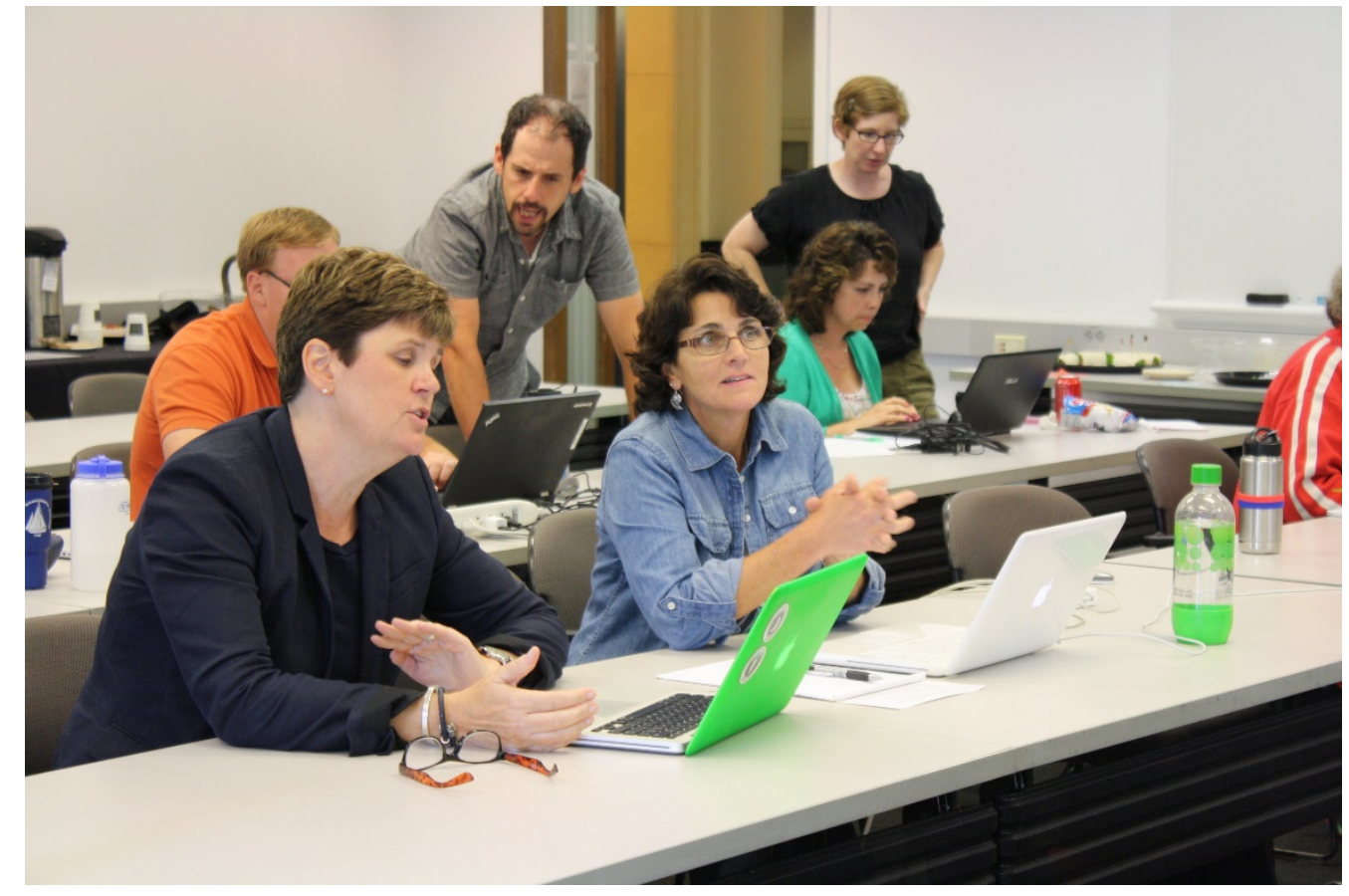

iDigPaleo Teacher Workshop - Yale Peabody Museum July 20-21, 2015 
Critical analysis by the audience

- heading logic

- facets for querying

- navigation issues

- user experience

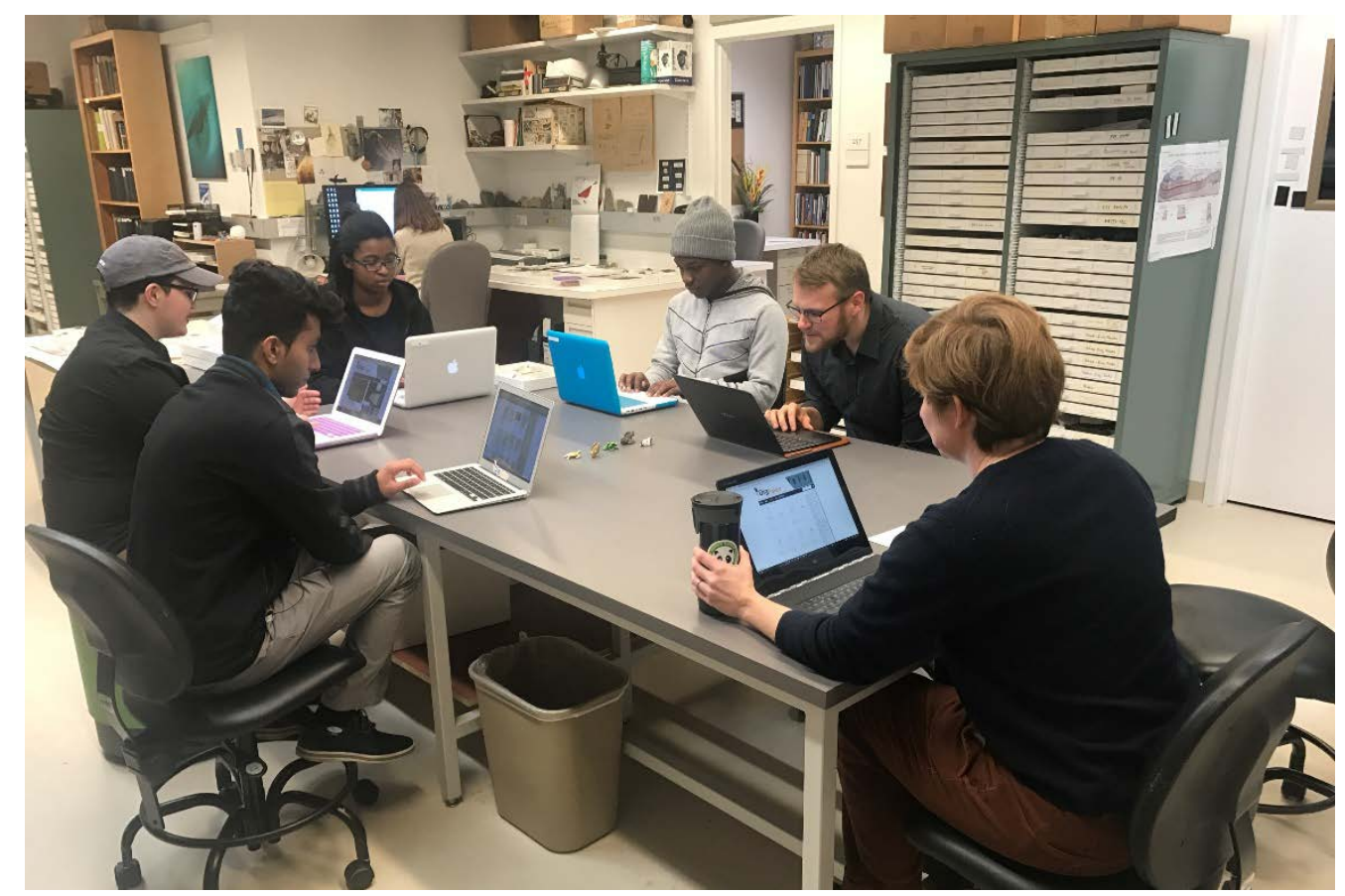

EVOLUTIONS high school interns - Yale Peabody Museum 2016-2017 


\section{Coming soon...}
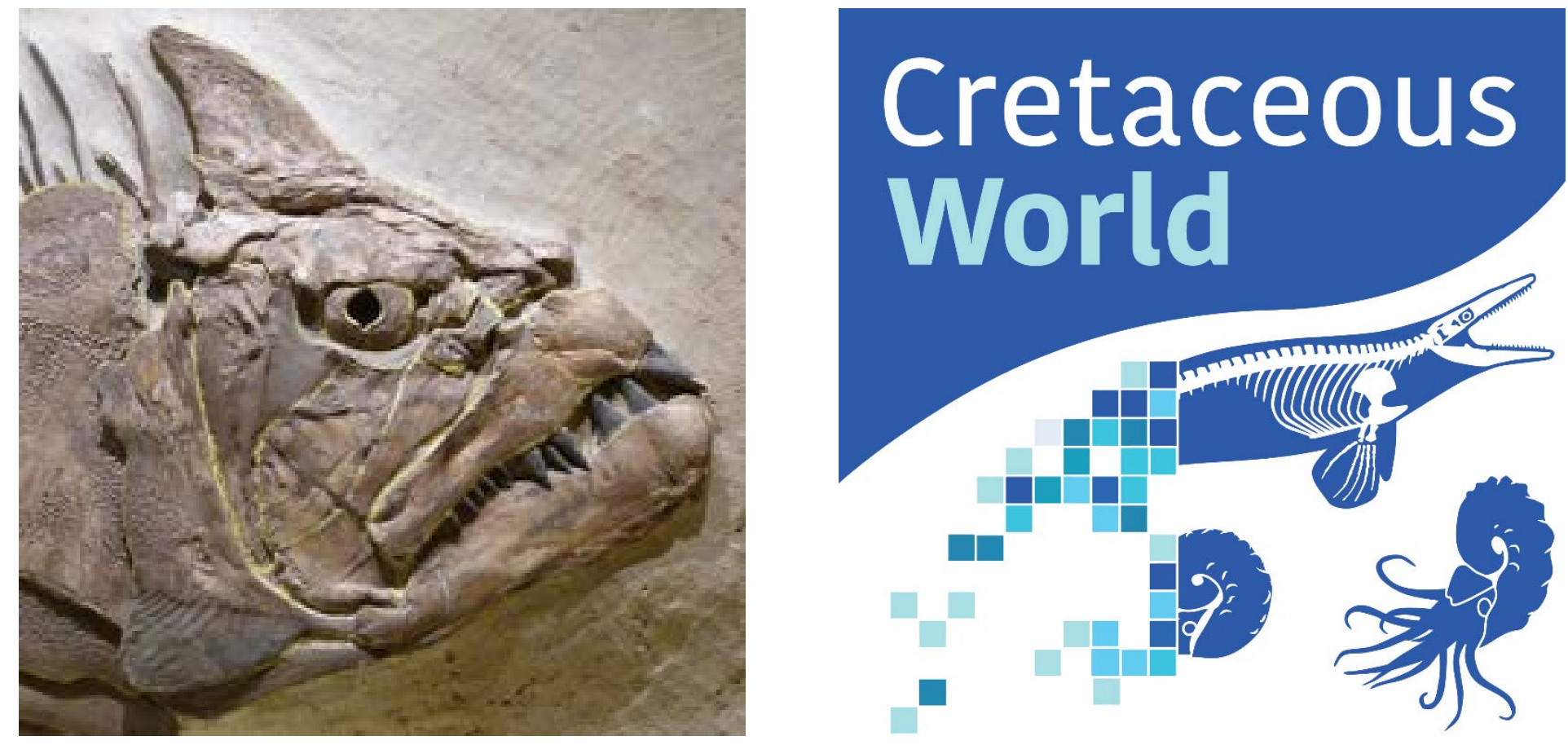

cretaceousworld.org 


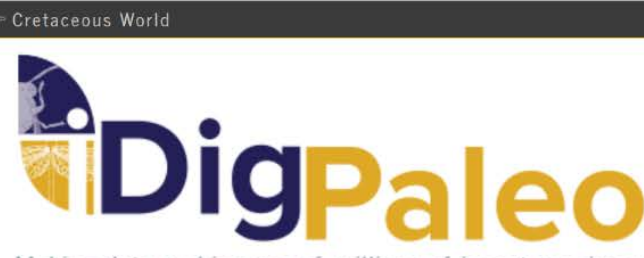

Making data and images of millions of insect specimens available on the web
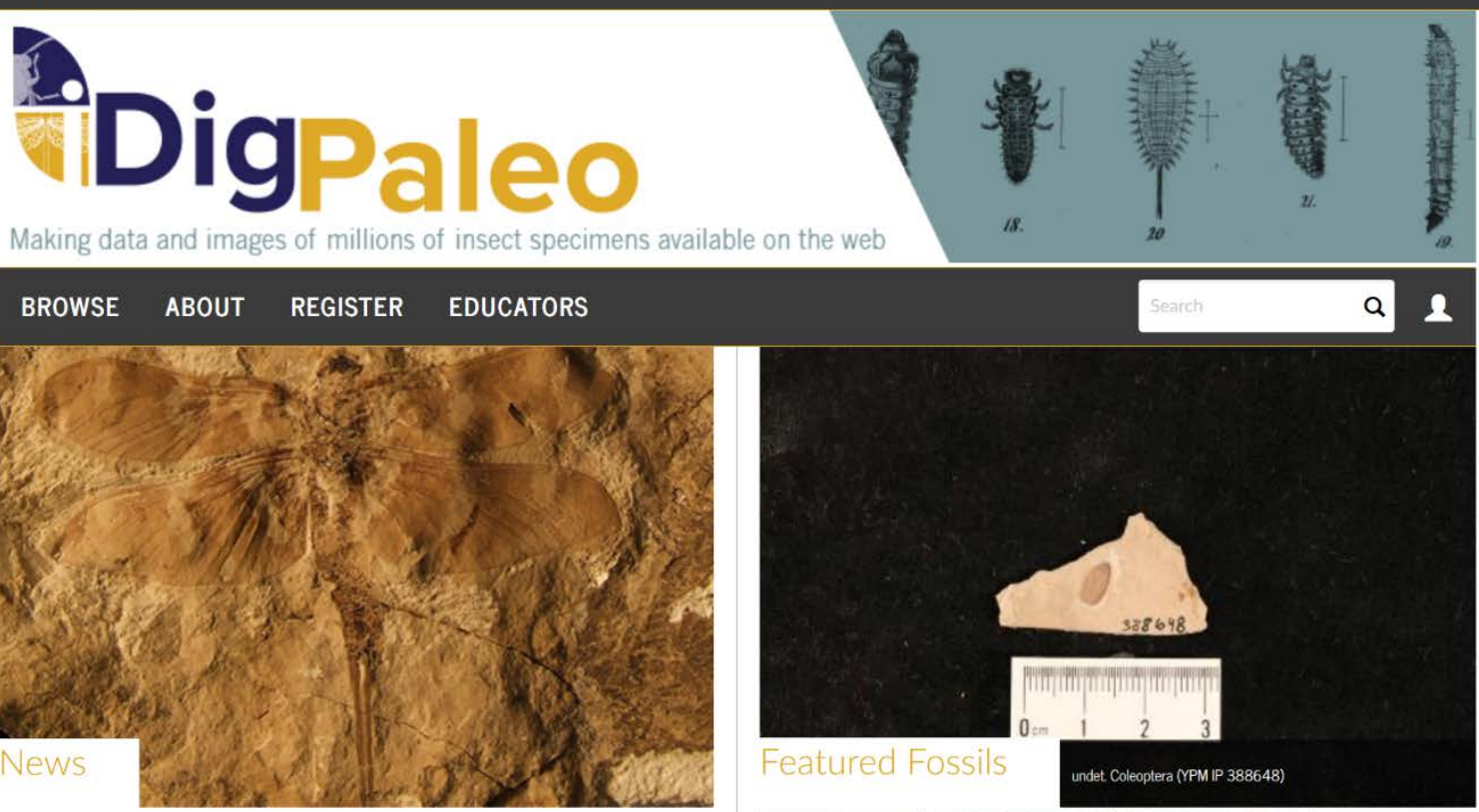

Fossil Insect Collaborative @ GSA 2016

More

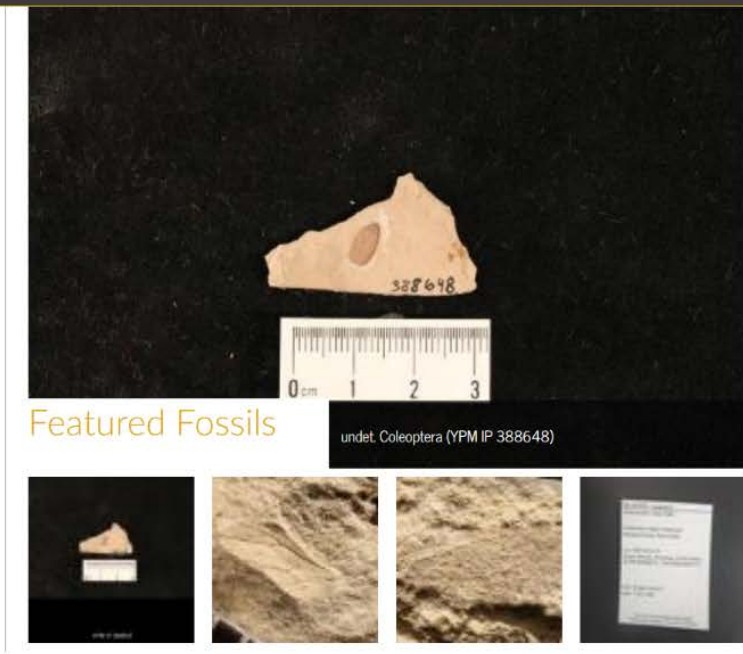

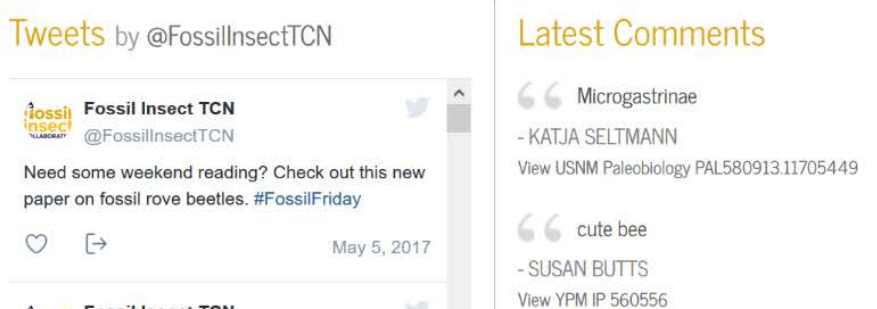

Featured Institution

Yale 

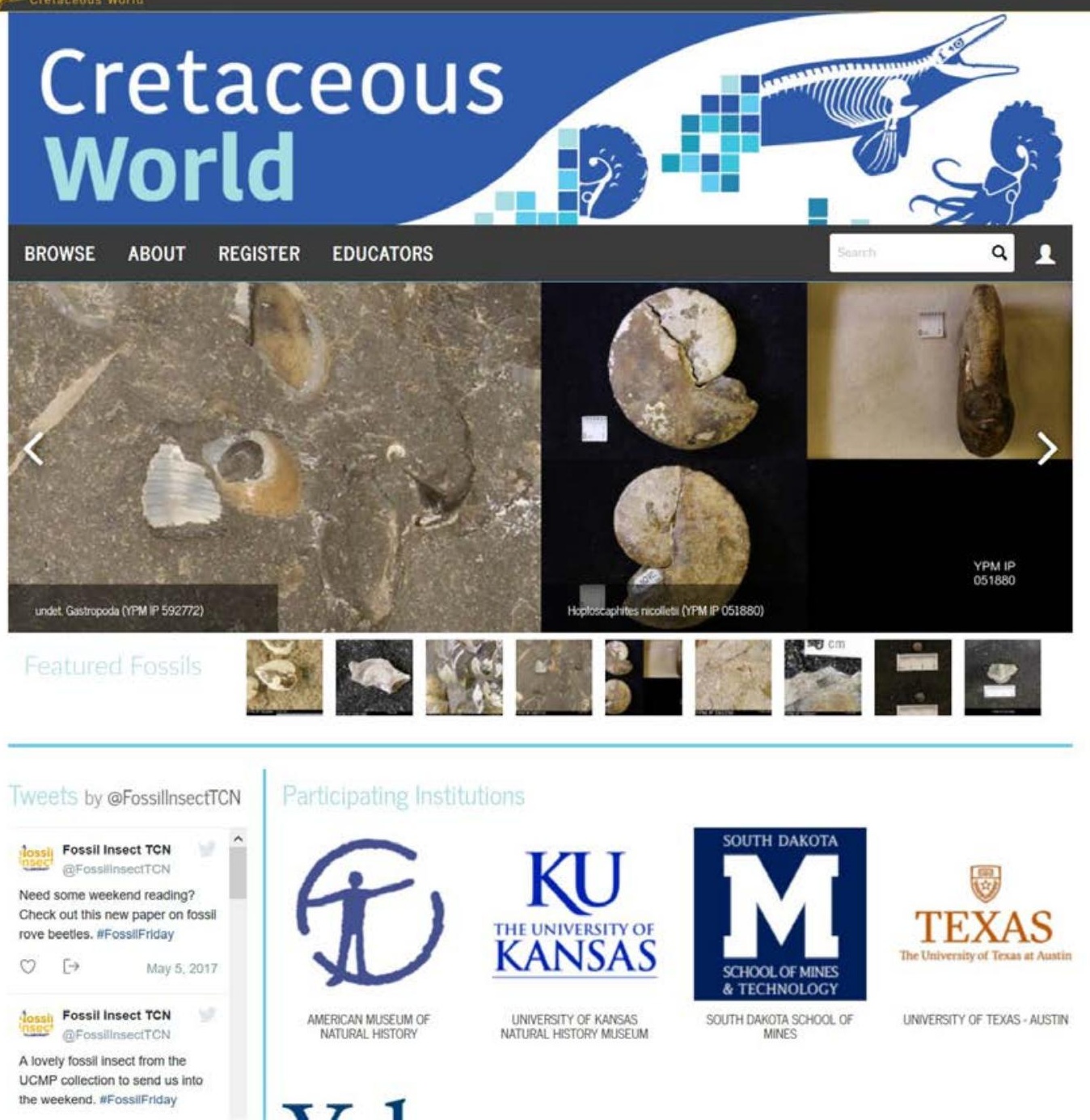
Cretaceous World

BROWSE ABOUT REGISTER EDUCATORS

249

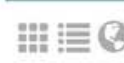

FILTER BY

HAS MEDUA HAS MEDIA $\odot$

COMMON NAME 6

AGE 10

STATE/PROVINCE 9

Genus 0

FOSSE DEPOST -1

source $\theta$
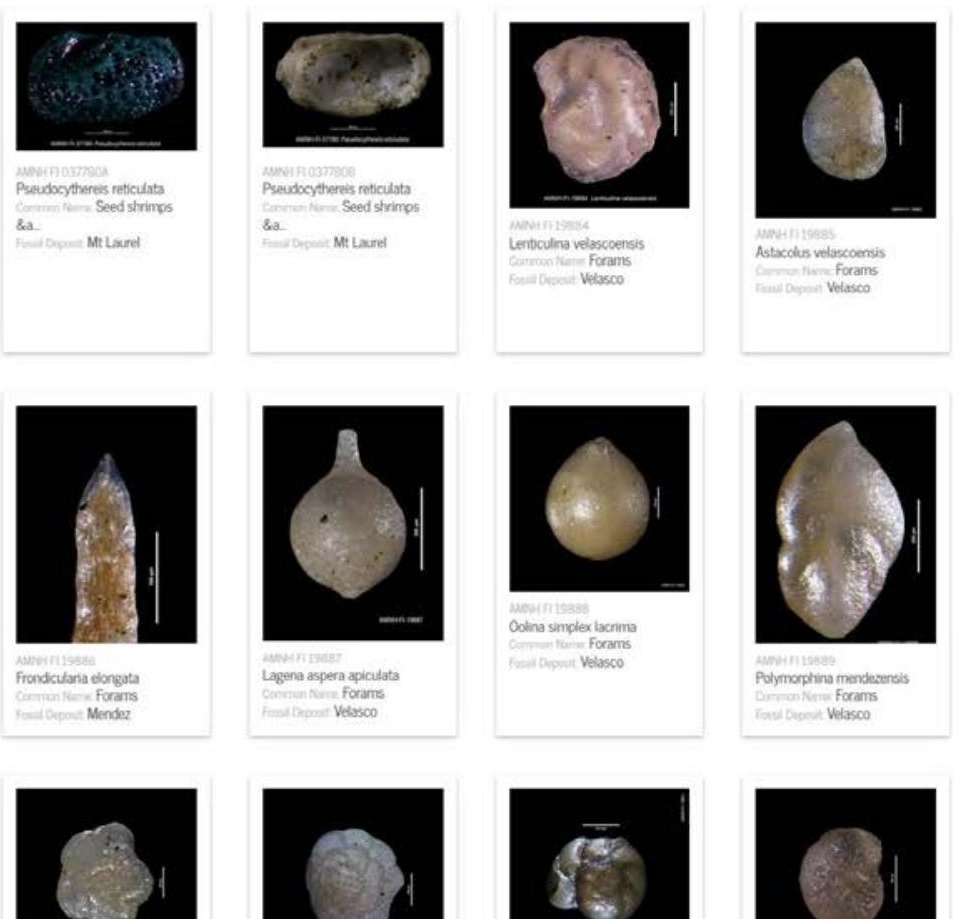


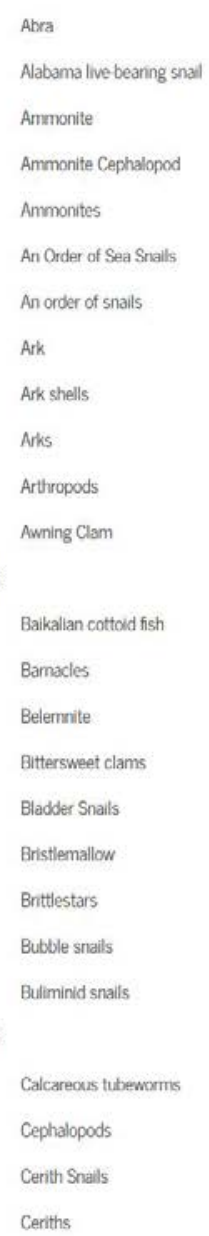

HAS MEDIA: HAS MEDIA O

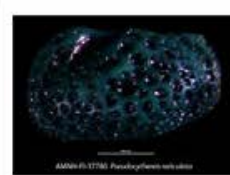

Pseudocytherels reticulata

Common Nanne Seed strimps

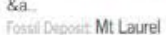
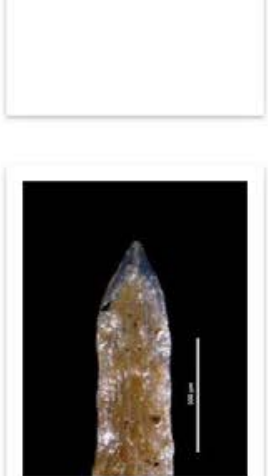

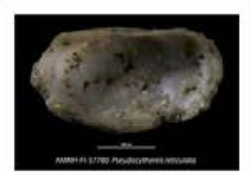

Pseurchoytheresis reticinat Common fame Seed shrimps \&a. Mi Laurel

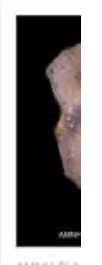

Lenticulir robse bap
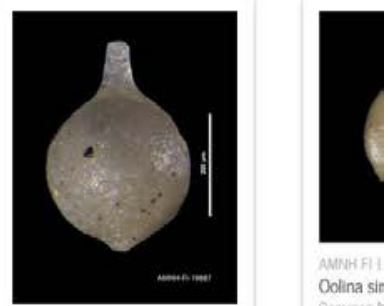


$$
\text { e }
$$



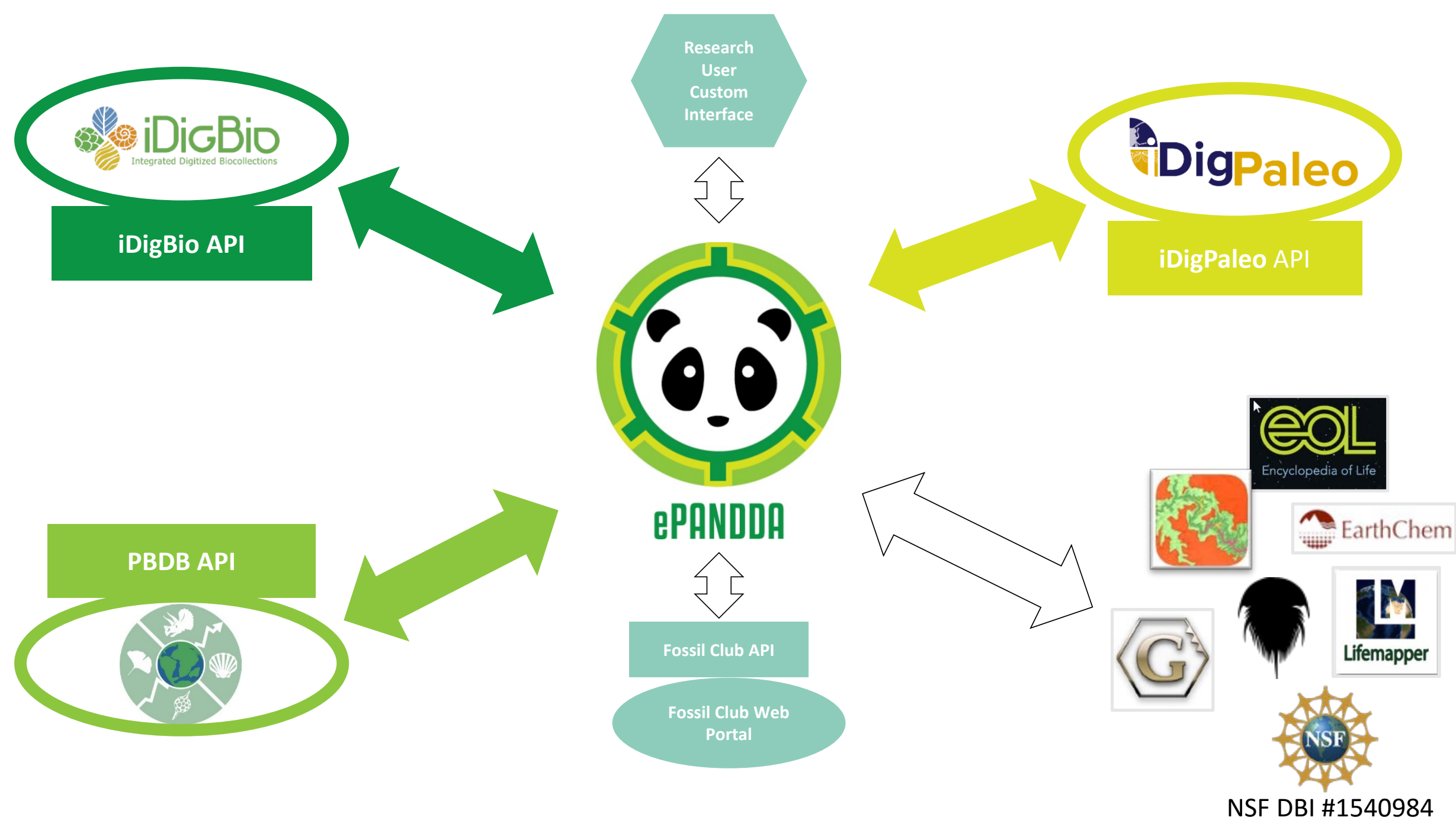


\section{Thank you}

Developer: Whirl-i-Gig, Seth Kaufman (Principal), Michael Benowitz, and others

FIC TCN PIs: Talia Karim (Lead), Dena Smith, David Grimaldi, Brian Farrell, Sam Heads, Michael Engel, Alton Dooley, Susan Butts, Chris Norris, and Diane Erwin

Cretaceous World TCN PIs: Bruce Lieberman (Lead), James Beach, K. Christopher Beard, Neil Landman, Laura Wilson, Jonathan Hendricks, Laurie Anderson, Maribeth Price, Talia Karim, Corinne Myers, Ann Molineux, Rowan Martindale, Matthew Brown, Susan Butts, and Christopher Norris

Collaboration with ePANDDA, iDigBio, Gary Motz (PaleoNiches PEN), and Florissant Fossil Beds NM 